\title{
The Role of Fetal Kidney Length and Fetal Transverse Diameter of Heart in Prediction of Gestational Age
}

\author{
Panda Subrat ${ }^{1}$, Rudrapal Gouri Sankar ${ }^{2}$, Das Ananya ${ }^{3}$, Ray Baruah Surajit ${ }^{4}$, Sharma Nalini ${ }^{3}$ \\ ${ }^{1}$ Associate Professor, ${ }^{2}$ Senior Resident, ${ }^{3}$ Assistant Professor, ${ }^{4}$ Academic Resident, \\ Department of Obstetrics \& Gynaecology, NEIGRIHMS
}

Corresponding Author: Panda Subrat; subrtpanda@gmail.com

Received 05 March 2020;

Accepted 26 March 2020;

Published 10 April 2020

\begin{abstract}
Objective: Currently there is no single ultrasonological parameter for accurate estimation of gestational age in third trimester. The present study is undertaken to validate the fetal kidney length and fetal transverse diameter of heart measurement as a measurement of fetal growth with less variability. Materials \& Methods: It is a cohort study conducted in Department of Obstetrics and Gynaecology, NEIGRIHMS from 2015 to 2016. 224 women with singleton pregnancies and sure of LMP were enrolled. Each woman underwent minimum three ultrasonographic measurements- at first trimester for gestational age by CRL, at second trimester with fetal conventional biometry, at third trimester with Fetal kidney length, transverse diameter of heart and fetal conventional biometric parameters and analysed with SPSS Version22. Results: The gestational age with CRL was within three days of the menstrual age. In second trimester, conventional parameter (BPD,HC,AC,FL) were better parameters. Conventional parameter did not correspond to gestational age calculated from LMP. (BPD, $R^{2}=0.4819, H_{C} R^{2}-0.507, A C R^{2}-$ 0.5175 , FL $R^{2}-0.5243$, Combined Conventional parameter $\left.R^{2}-0.5888\right)$. Fetal kidney length $\left(R^{2}-0.8356\right)$ and transverse diameter of heart $\left(R^{2}\right.$ $0.8795)$ better correlated with gestational age. Conclusion: Kidney length and transverse diameter of heart are better predictors of gestational age in third trimester.
\end{abstract}

Keywords: Estimation of gestational age; Conventional parameters; fetal kidney length; fetal transverse diameter of heart

\section{Introduction}

An accurate determination of the gestational age and the expected date of delivery is fundamental to the successful management of all pregnancies. Initiation of Janani Surakshya Yojana has made an increase in the trend of institutional delivery in India ${ }^{[1]}$. As a result, a good number of high risk pregnancies attend the hospitals for the first time in the third trimester. This poses a challenge to the obstetrician as the difficulty here is when to terminate the pregnancy in the absence of accurate LMP and early trimester scans. This scenario is made worse in Intra Uterine Growth Restricted Fetuses (IUGR) where there is a huge discrepancy in gestational age by last menstrual period and per abdominal obstetric finding or even worse when the lady is not sure of her last menstrual period.. Utilizing ultrasound to visualize different fetal anatomical landmarks and to follow their growth during the ante natal check up is one of the most important Obstetric tools for the evaluation of the fetus ${ }^{[2]}$. Estimating gestational age from an early measurement of crown rump length in first trimester is accurate to within three days ${ }^{[3]}$ and multiple biometric parameters in third trimester is less accurate to within three weeks. The sonographic parameters used in the third trimester are fetal abdominal circumference, biparietal diameter and femur length. Currently there is no available third trimester ultrasound parameter which can predict gestational age accurately and one such parameter which remains unaltered even by Intra Uterine Growth Restriction (IUGR) will solve the therapeutic dilemma of the obstetrician and nullify the probability of iatrogenic prematurity. Determination of gestational age by ultrasound has similar accuracy when performed between 11-14 weeks of gestation and 18-22 weeks of gestation with increase in margin of error after 22 weeks. ${ }^{[4]}$ As gestational age progress, they become increasingly unreliable because of the biological variability of size in relation to age. Currently there is no single fetal measurement used for accurate estimation of gestational age in the third trimester especially in women who booked late and unsure about their LMPs. The present study is undertaken to validate the fetal kidney length and fetal transverse diameter of heart measurement as an additional morphological parameter of fetal growth with less variability

Materials and methods: It is a prospective cohort study carried out in the Department of Obstetrics and Gynaecology, North Eastern Indira Gandhi Regional Institute of Medical Sciences (NEIGRIHMS) during the period January 2014 to June 2015 with the help of Department of Radiology NEIGRIHMS. There were 224 women with singleton pregnancies, conceived 
spontaneously and sure of the dates of their last normal menstrual period were enrolled for the study. Unknown or inaccurate date of last menstrual period, pregnant women with irregular menstrual cycles, multifetal gestations, fetal chromosomal abnormalities, fetal anomaly, cardio thoracic ratio greater than 0.50 , polyhydramnios, oligohydramnios and renal pelvic dilatation of $5 \mathrm{~mm}$ or greater were excluded from the study. Each woman underwent minimum three ultrasonographic measurements: first trimester for ascertaining gestational age by measuring crown rump length, next at second trimester along with fetal anomaly scan, fetal biometry with fetal kidney length and fetal transverse diameter of heart measurement. And finally at third trimester along with fetal biometric measurement (Bi Parietal diameter, head circumference, Abdominal Circumference, Femur Length) transverse diameter of heart and kidney length were taken.

Kidney Length measurement were obtained in the sagittal plane, when full length of kidney with renal pelvis is visualized. Maximum length of anyone single fetal kidney is measured from upper pole to lower pole at least thrice and mean of the measurements is taken. Usually, renal length measurement in the fetus increase with gestational age. A "rule of thumb" measurement is that the menstrual age in weeks approximates the normal fetal kidney length in millimeters. The same was applied for the Transverse Diameter of Heart as well.

If the difference in gestational age from Last Menstrual Period and Crown Rump Length measurement is less than 7 days, the gestational age is based on Last Menstrual Period only. If the difference is more than 7 days, the gestational age is based on the
Crown Rump Length measurement. This gestational age was taken as the actual gestational age for further assessment and analysis (by Last Menstrual Period or by Crown Rump Length whichever was more accurate.)

The obtained data were recorded in a Microsoft Excel Sheet to produce the Master chart for statistical analysis. The data were analysed using the software "Graphpad Prism 6". First Bivariate Correlation was analysed to obtain Pearson Correlation coefficient. Secondly, Linear Regression Analysis was performed to obtain the Slope, Y intercept, 95\% confidence interval, Standard error of Prediction and $\mathrm{p}$ value.

\section{Results \& Observations}

In the first trimester, there is significant correlation of estimation of gestational age by CRL measurement and menstrual age with $\mathrm{R}^{2}$ $0.877, \mathrm{p}$ value $<0.0001$ and +-3 days of variation. In the second trimester, ultrasonographical parameter BPD,HC,AC,FL were corresponding with gestational age calculated from LMP. $\left(\mathrm{R}^{2}\right.$ $0.9203,0.9256,0.91370 .9369)$, and better accuracy +-5to7 days with the menstrual age. The correlation of kidney length and transverse diameter of heart was found to be $\mathrm{R}^{2} 08357$ and 0.8354 . Kidney length and Transverse diameter of the heart are more accurate when gestational age was calculated from menstrual age $\left(\mathrm{R}^{2} 0.8356\right.$ and 0.8795$)$ than conventional parameters like BPD $\left(\mathrm{R}^{2}\right.$ $0.4819)$, HC $\left(R^{2} 0.5070\right) A C\left(R^{2} 0.5175\right)$ FL $\left(R^{2} 0.5243\right)$ and combined conventional parameter $\left(\mathrm{R}^{2} 0.5885\right)$ and with variation of +-11 to 12 days with the menstrual age.

Fig 1: Correlation of different fetal parameters with gestational age.

\begin{tabular}{|c|c|c|c|c|c|c|c|}
\hline Trimester & Parameter & Slope & $\mathbf{R}^{2}$ & $\begin{array}{l}\text { 95\% Confidence } \\
\text { interval }\end{array}$ & $Y$ intercept & $\begin{array}{l}\text { Standard error } \\
\text { of prediction }\end{array}$ & $\begin{array}{l}\mathbf{p} \\
\text { value }\end{array}$ \\
\hline First & CRL & $0.8642+-0.02198$ & 0.8744 & 0.9163 to 0.9498 & $7.878+-1.480$ & 3.636 & $<0.0001$ \\
\hline \multirow[t]{6}{*}{ Second } & BPD & $1 . \mathrm{O} 29+-0.02032$ & 0.9203 & 0.9474 to0.9686 & $-3.929+-3.006$ & 6.016 & $<0.001$ \\
\hline & $\mathrm{HC}$ & $0.9749+-0.018556$ & 0.9256 & 0.9509 TO 0.9707 & $2.617+-2.745$ & 5.493 & $<0.0001$ \\
\hline & $\mathrm{AC}$ & $0.9941+-0.02050$ & 0.9137 & 0.9430 to0.9659 & $-1.282+-3.033$ & 6.069 & $<0.0001$ \\
\hline & FL & $0.9914+-0.01727$ & 0.9369 & 0.9585 to 0.9753 & $-0.9914+-2.554$ & 5.111 & $<0.0001$ \\
\hline & Kidney length & $1.093+-0.03255$ & 0.8357 & 0.8897 to0.9334 & $-0.03694+-0.6827$ & 1.409 & $<0.0001$ \\
\hline & $\begin{array}{l}\text { Heart Transverse } \\
\text { diameter }\end{array}$ & $1.494+-0.04450$ & 0.8354 & 0.8895 to 0.9333 & $-12.78+-0.9334$ & 1.927 & $<0.0001$ \\
\hline \multirow[t]{7}{*}{ Third } & BPD & $0.5393+-0.03753$ & 0.4819 & 0.6195 to 0.7564 & $106.5+-9.653$ & 11.34 & $<0.0001$ \\
\hline & $\mathrm{HC}$ & $0.5665+-0.03749$ & 0.5070 & $0.6408 \mathrm{t} 00.7712$ & $95.96+-9.641$ & 11.32 & $<0.0001$ \\
\hline & $\mathrm{AC}$ & $0.6048+-0.3919$ & 0.5175 & 0.6496 to 0.7772 & $84.14+-10.08$ & 11.84 & $<0001$ \\
\hline & FL & $0.6556+-0.04191$ & 0.5243 & 0.6552 to 0.0 .7810 & $70.80+\_10.78$ & 12.66 & $<0.0001$ \\
\hline & $\begin{array}{l}\text { Combined } \\
\text { conventional }\end{array}$ & $0.5863+-0.03288$ & 0.5888 & 0.7074 to0.8163 & $89.74+-8.457$ & 9.932 & $<0.0001$ \\
\hline & Kidney length & $0.7958+-0.03366$ & 0.8356 & 0.8773 to 0.9402 & $7.780+-1.232$ & 1.020 & $<0.0001$ \\
\hline & $\begin{array}{l}\text { Heart Transverse } \\
\text { diameter }\end{array}$ & $1.033+-0.0$ & 0.8795 & 0.9107 to 0.9569 & $-1.033+-0.03645$ & 1.104 & $<0.001$ \\
\hline
\end{tabular}

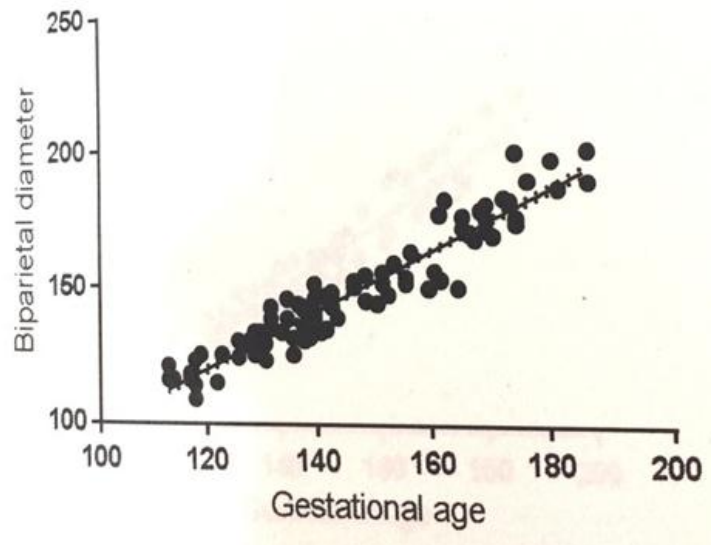

Fig 2: Correlation of BPD and gestational age in $3^{\text {rd }}$ trimester.
Correlation Head Circumference

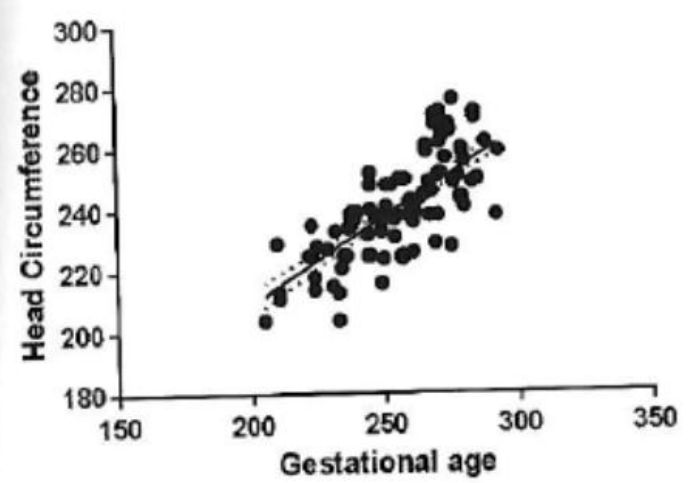

Fig 3: Correlation of $\mathrm{HC}$ and gestational age in $3^{\text {rd }}$ trimester 


\section{Correlation- Femur Length}

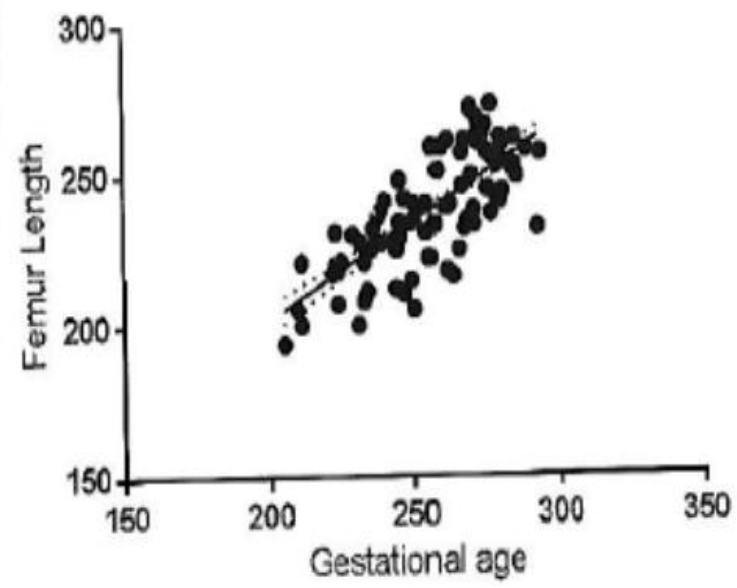

Fig 4: Correlation of FL and gestational age in $3^{\text {rd }}$ trimester

\section{Correlation- Comibned}

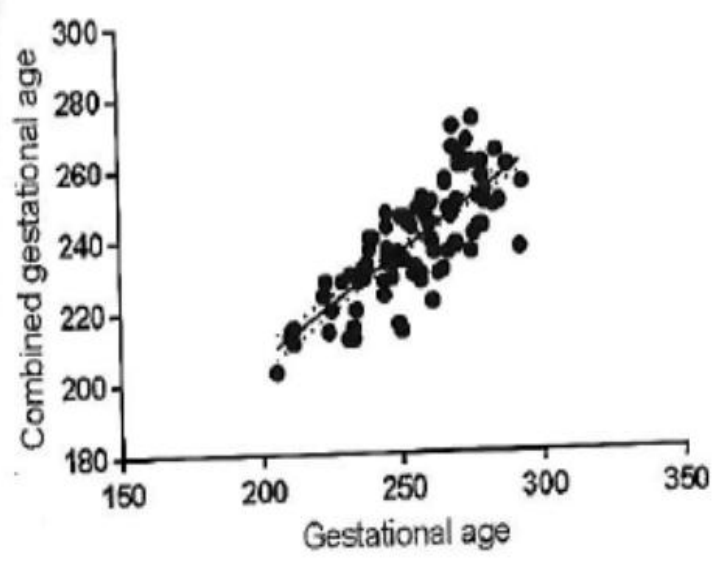

Fig 5: Correlation of combined ultrasound parameters with gestational age in $3^{\text {rd }}$ trimester.

\section{Correlation- Kidney Length}

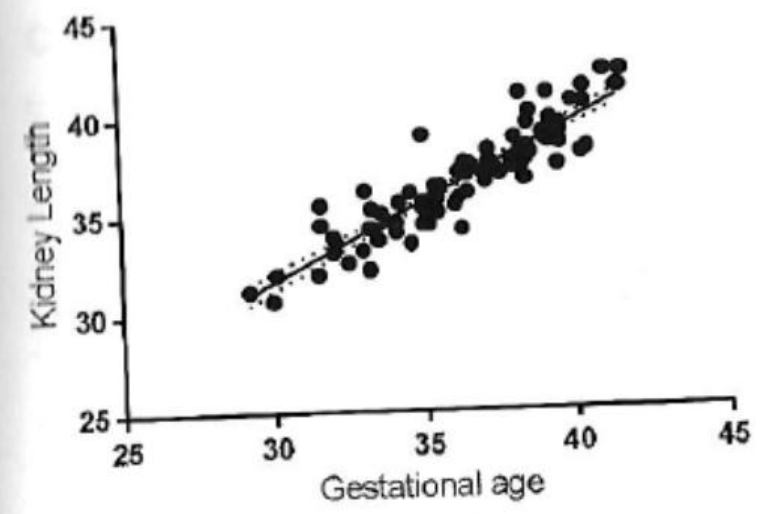

Fig 6: Correlation of fetal kidney length with gestational age.

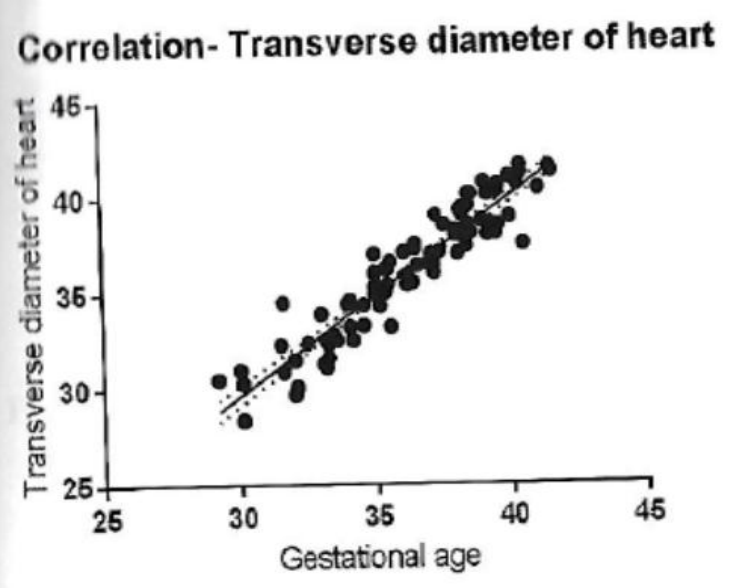

Fig 7: Correlation of fetal transverse diameter of heart with gestational age.

\section{Discussion}

The Ultrasonic measurement of fetal Crown-Rump Length as a method of assessing gestational age was found to be within three days of the menstrual age. In the third trimester conventional parameters (BPD, HC, AC and FL) did not correspond to the gestational age calculated from LMP. Fetal kidney length and transverse diameter of the heart better correlated with the gestational age calculated from LMP. Even combined conventional parameter $(\mathrm{BPD}+\mathrm{HC}+\mathrm{AC}+\mathrm{FL})$ did not estimate the gestational age.

L.J.Saloman ${ }^{[5]}$ et al. conducted a study on "Revisiting firsttrimester fetal biometry" and concluded that using CRL as predictor, the $95 \%$ prediction interval and the standard error (SE) of estimates were +2.44 and correlation coefficient being 0.87 which is nearly similar to findings of our study.

In the second trimester $\mathrm{BPD}, \mathrm{HC}, \mathrm{AC}, \mathrm{FL}$ usually corresponds to the menstrual age and is better predictor of gestational age than kidney length and transverse diameter of the heart. F P Hadlock et al. conducted the study on "Fetal biparietal diameter: a critical re-evaluation of the relation to menstrual age by means of real-time ultrasound and concluded that the variability associated with predicting menstrual age from the BPD increased progressively throughout gestation; the maximal variability was noted between 36 and 42 weeks (+/- 3.6 weeks) with $R^{2}$ value of 0.98 . Our study findings are similar to the observation. Hadlock F. P. et al. conducted the study on "Fetal head circumference: relation to menstrual age" and concluded that mathematical modeling of the data demonstrated that the head circumference growth curve is nonlinear, similar to the bi parietal diameter growth curve. In the study predicted menstrual age value (=/- $2 \mathrm{SD}$ ) associates with a given head circumference measurement were calculated and are presented in tabular form. The $\mathrm{R}^{2}$ of the study was $98.3 \%$. .Our study results are similar to the above mentioned study. C B Benson and P M Doubilet ${ }^{[6]}$ conducted a study titled "Sonographic prediction of gestational age: accuracy of second - and third trimester fetal measurement". They found that the accuracy of all predictors diameter and head circumference were more accurate predictors of gestational age than were bi parietal diameter, femoral length, and abdominal circumference. In our study Head Circumference was almost of similar accuracy compared to other mentioned parameters in predicting gestational age. F P. Hadlock et al. conducted the study on "Fetal Abdominal Circumference as a predicts of Menstural Age" and observed that abdominal Circumference predicts the gestational age accurately within +1.49 weeks between $12-18$ weeks of gestation, +2 weeks between $18-24$ 
weeks of gestation ${ }^{[7]}$. The $\mathrm{R}^{2}$ of the study was $97.9 \%$. Our finding is within the range of the study. Warda, Ahmed H. et al. conducted a study on Fetal Femur Length: a critical reevaluation of the relationship to Menstural Age and observed that gestational age predicted by femur length measurement has variability (in the upper range) of $+1.4-1.9$ weeks from 12 to 1 weeks, $+1.9 \_2.6$ weeks from 18 to 24 weeks $^{[8]}$. The $\mathrm{R}^{2}$ was $96.8 \%$. Our study finding is again within the range of the above observation.

In our study Femur Length measured in the third trimester predicted the gestational age accurately within +-12.66 days which was poorly correlated. $\left(\mathrm{R}^{2} 0.5243\right)$

F P Harlock, R B Harrist, R L Deter and S K Park conducted another study on "A prospective of fetal femur length as a predictor of gestational age" and concluded that "Variability increased throughout pregnancy, ranging from +/- 11.6 days57 between 18 and 24 weeks to $+/-22.7$ days in the last six weeks pregnancy" ${ }^{\text {"[9] }}$. In our study combined ultrasound age ( by combining Bi parietal diameter, Head Circumference, abdominal circumference and femur length in the third trimester predicted the gestational age accurately within +-9.9 days which had poor correlation with the gestational age $\left(\mathrm{R}^{2} 0.5888\right)$

Frank P. Hadloch et al. conducted the study on" Fetal Abdominal Circumference as a predictor of mensural age" ${ }^{\text {"[10] }}$. They suggested that biparietal diameter, head circumference, abdominal circumference and femur length combination of parameter was more accurate in predicting menstrual age than any single parameter, particularly in the third trimester of pregnancy. Our study finding is similar to the above finding.

Babuta, Sumit, et al. conducted a study on "Assessment of fetal gestational age in different trimesters from ultrasonographic measurements of various fetal biometric parameters" and observed that biparietal diameter and head circumference were found to be equally good predictors of gestational age and to determine the expected date of delivery (EDD) in the second trimester and BPD and FL in the third trimester ${ }^{[11]}$. They also observed that accuracy of predicting gestational age was +-2 to 4 weeks in the third trimester.

Nirmala Shivalingaiah et al. conducted a study on "Fetal Kidney length as a parameter for determination of gestational age in pregnancy" and observed that the mean deviation from the gestational age at all the weeks is least for Kidney Length which correlated well with the assigned gestational age and found almost same as all the ultrasound biometric parameters put together ${ }^{[12]}$. Our study observation is similar to this study except that in third trimester of pregnancy Kidney Length measured gestational age more accurately than the conventional parameters. Foetal. Transverse Diameter of Heart measured in the second trimester showed correlation $\left(\mathrm{R}^{2} 0.8354\right)$ with gestational age predicted the age accurately within +-1.9 weeks. When measured in the third trimester transverse Diameter of the Heart showed correlation $\left(\mathrm{R}^{2}\right.$ $0.8795)$ with gestational age predicting it accurately within +-1.1 week.

Bertagnoli et al. in their study confirmed that fetal kidney measurements can be used in the routine assessment of gestational age which matches with my study purpose and observation. ${ }^{[12]}$

Haritha Kumari. $\mathrm{N}$ et al. in their study showed that there was a significant growth of fetal Heart Diameter from age group 20-24 weeks and 32-36 weeks compared to rest of the gestational weeks. The data obtained was presented in tables (Diameter of heart in relation to gestational age). ${ }^{[13]}$

\section{Conclusion}

Precise knowledge of gestational age is the key to the successful management of pregnancy. Fetal Kidney length and transverse diameter of fetal heart are better predictors of gestational age in third trimester than combined ultrasound age (biparietal diameter, head circumference, abdominal circumference and femur length). This concept is especially useful for the management of increasing number of unbooked pregnant women coming for institutional deliveries with no antenatal checkup and previous ultrasound report. But, to expand the utility of the concept, further study is required to establish the role of those two parameters in predicting the gestational age in small for gestational age pregnancies.

\section{Conflict of Interest}

None

\section{References}

[1] Hertz RH, Sokol RJ, Knoke JD et al. Clinical estimation of gestational age: Rules for avoiding preterm delivery. Am J Obstet Gynecol1978; 131: 395.

[2] Robinson HP, Fleming JE. A critical evaluation of sonar "crown-rump length" measurements $\mathrm{Br} \quad \mathrm{J}$ Obstet Gynaecol. 1975 Sep;82 (9):702-10.

[3] Ballard JL, Novak KK, Driver M. A simplified score for assessment of fetal maturation of newly born infants. J Pediatr. 1979 Nov;95 (5 Pt 1):769-74.

[4] Olsen, O, Clausen, JA. Routine ultrasound dating has not been shown to be more accurate than the calendar method. Br J Obstet Gynaecol 1997; 107: 1221- 1222.

[5] Boerhaave, H. Praelectiones Academicae in Proprias Institutiones Rei Medicae. A Haller, editor. Amstelaedami: Wetstenium, 1744: 437.

[6] Naegele, FC. Erfahrungen und Abhandlungen aus dem Gebiethe der Krankheiten des Weiblichen Geschlechtes. Nebst Grundzügen einer Methodenlehre der Geburtshülfe. Mannheim: Loeffler, 1812:280- 281.

[7] Speert, H. Obstetric and Gynecologic Milestones. New York: The Parthenon Publishing Group Ltd, 1996: 171172.

[8] Bedford, GS. The Principles and Practice of Obstetrics. 5th Edition. New York: William Wood and Co, 1872: 306.

[9] Cock, TF. A Manual of Obstetrics. New York: Samuel S, Wm. Wood, 1853: 76.

[10] Rosati P, Guariglia L. Transvaginal sonographic assessment of the fetal urinary tract in early pregnancy. Ultrasound Obstet Gynecol. 1996 Feb; 7(2):95-100.

[11] Rossavik IK, Torjusen GO, Gibbons WE Conceptual age and ultrasound measurements of gestational sac and crown-rump length in vitro fertilization pregnancies. Fertil Steril. 1988 Jun; 49(6):1012-7.

[12] Bertagnoli L, Lalatta F, Gallicchio R, Fantuzzi M, Rusca M, Zorzoli A, Deter RL. Quantitative characterization of the growth of the fetal kidney. J Clin Ultrasound. 1983 Sep; 11(7):349-56.

[13] Haritha Kumari.N, Aruna Mukherjee,B.K.Mathur. Measurement Of Fetal Heart Dimensions At Different Stages Of Development In Indian Population Of Maharashtra Region, International Journal Of Collaborative Research On Internal Medicine \& Public Health 